\title{
Advanced design and characterization methodologies for memory-aware CMOS power-amplifier implementation
}

\author{
Martin Schleyer, Dominic Maurath, Heinrich Klar, and Friedel Gerfers \\ Department Mixed Signal Circuit Design, Faculty IV, Technical University Berlin, 10587 Berlin, Germany \\ Correspondence to: Dominic Maurath (maurath@tu-berlin.de)
}

Received: 21 December 2016 - Revised: 13 April 2017 - Accepted: 22 April 2017 - Published: 21 September 2017

\begin{abstract}
This paper reports on an effective root-cause analysis method of memory effects in power amplifiers, as well as introduces compensation techniques on a circuit design level. Despite conventional memory-effect approaches, the discussed method uses a two-tone scan over a wide operation and modulation range. This enables an in-depth study of physical causes and helps to implement compensation techniques at design stage. On the one hand, this circuit investigation is optimized using an automated SystemC model parametrized with real device and measurement values. Hence, computation time is widely reduced which shortens design cycles. On the other hand, the implementation of the derived circuit compensation means will reduce the complexity of digital pre-distortion due to a reduced memory-effect induced AM/AM and AM/PM hysteresis. The approach is demonstrated on a $65 \mathrm{~nm}$ CMOS power amplifier with an OIP1 of $27 \mathrm{dBm}$ and a PAE of over $30 \%$ using WCDMA and LTE signals. In fact, mismatch could be reduced by more than $8 \%$.
\end{abstract}

\section{Introduction}

A common approach in radio transmitter design is using digital pre-distortion (DPD) and linearization in order to deal with power amplifier induced non-linearity. These nonlinearities are usually a function of signal power and frequency. Since such effects are very deterministic, DPD can be used with high reliability. However, as real transmitter systems are actually more complex the signal history has appeared as an important measure for signal distortion (Bösch et al., 1989). This is addressed/concluded as the memory effect of power amplifiers, and is non-deterministic (no (quasi) steady state available) due to the unknown signal history at design time (Martens et al., 2012). That is, while distortion caused by regular non-linearity (frequency, amplitude) is much easier to be compensated, non-linearity induced by memory effects inflate DPD efforts, causing significant processing overhead, and reduce the reliability of RF signal fidelity (Brunk et al., 2011). That is, in non-linear circuits there are intermodulation products near the location of the actual modulation frequencies and its harmonics (Vuolevi et al., 2000). In case of further non-linear processes the impedances of the source, load, and the amplifier itself become more important as the modulation frequencies are mixed into the band of the fundamental frequency.

As the need for higher data rates and spectrum efficiencies continuously rises, so is the need for RF signal linearity. Higher-order modulation schemes only allow a small error vector magnitude, and thus, any amplitude or phase error directly degrades possible data rate, i.e. increases the bit-error rate (BER). Further on, power amplifier memory effects usually causes asymmetric sidebands in the frequency spectrum, which is problematic for the needed compliance to adjacent channel power ratio specifications (e.g. $-60 \mathrm{dBm})-$ hence, the maximum output power of the radio transmitter need to be artificially reduced.

As shown in Fig. 1a, the measurement setup not only includes a signal source (waveform generator), DC sources and a scope, but also uses a MATLAB controller. The advantage of the MATLAB controller is that amplitude and frequency ranges can be better adjusted, and the data processing is direct and centrally organized in one element. The trigger signal is to automatically step the two-tone frequency and amplitude over the complete range. The two-tone approach is introduced by Wolf et al. (2010) as an efficient method to scan the thermal and electrical memory effects over a large input power range. 

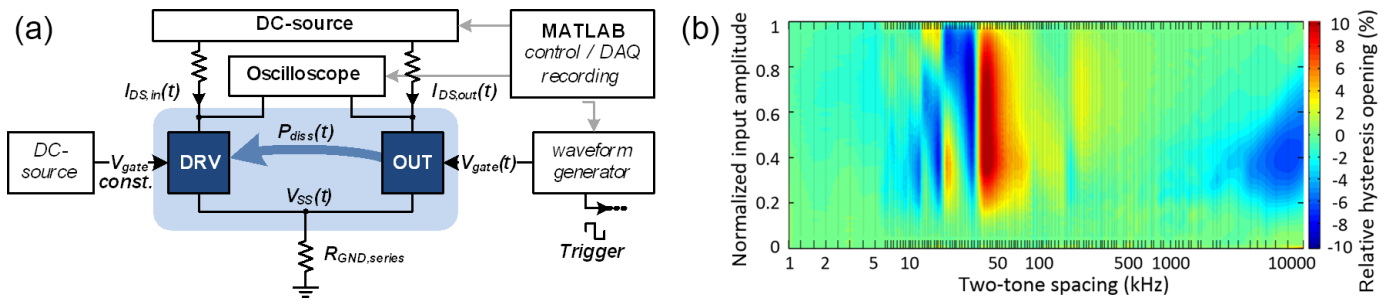

Figure 1. Measurement setup and heat map of a two-tone scan.
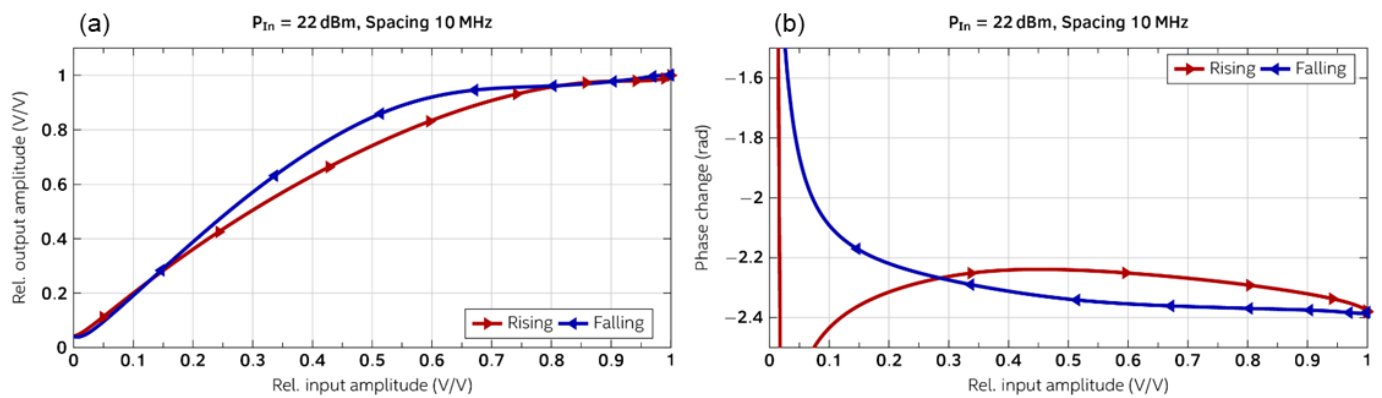

Figure 2. Evaluation of the time-domain measurement with $P_{\text {in }}=22 \mathrm{dBm}$ and $10 \mathrm{MHz}$ two-tone spacing.

In general, there are short-term, and long-term memory effects. Both have different physical root-causes as suggested in Wolf et al. (2010). Short-term effects are referred to as electrical effects (inductive behavior) usually caused by frequency depend impedances of the source, the load, and the amplifier itself. This is particular significant for narrowband matching networks. The long-term effects are caused by thermal processes due to thermal conductance and thermal capacitance (e.g. due to self-heating) Hence, transferring these electrical and thermal effects into a system design context, there are additional poles and zeros due to the memory effect. Figure $1 \mathrm{~b}$ provides a heatmap recorded with the twotone setup. While the left-handed areas refer to thermal effects (long-term, capacitive poles), the further right-hand side areas refer to a larger envelop frequency, thus, indicating memory effects related to electrical causes (short-term, inductive zeros). The red to blue color range indicates positive and negative distortions of the AM/AM time-domain trajectories. The sudden phase change at about $35 \mathrm{kHz}$ two-tone spacing indicates a thermal pole. As usually multi-stage amplifiers are used, there is a critical issue if the thermal coupling between individual amplifier stages create additional feedback loops which interact with the designed and implemented electrical feedback loops. That is, in conjunction with such thermal poles, the phase margin deteriorates, and the power amplifier could even enter instability, i.e. resonance states. Thus, causes signal loss, as well as device stress and operation failure.

Figure 2 shows a concrete sample of the heatmap scan. For better distinction, the plots use a blue line for ascending amplitudes, and a red line for decreasing amplitudes. More specifically, it can be concluded that the influence of the thermal RC network on AM/AM results for higher temperatures are that there is (i) less gain, and (ii) a frequency shift due to capacitive behavior. This is also clearly visible in the model as well as measurements. The relation of the time constants and hysteresis, is that there is for low frequencies also a low hysteresis, but no hysteresis for high frequencies. Furthermore, the simulation shows a strong hysteresis around corner frequency.

\section{Modelling, parameter extraction and design flow}

Figure 3, as well as Fig. 1 in (Schleyer et al., 2014), provide more insights into the SystemC/AMS time-domain model (timed data flow model) which is linked to the MATLAB controller. The advantage is that model parameters can be directly used from the measurement setup, such as balun specifications, and from the design files, such as the impedance matching network values (Spectre small signal results, Sparameter touchstone files, csv data extracted from measurements, circuit simulations, or system specifications). That is, parameter extraction from measurement results is done with MATLAB, the other model data are provided in XML. This reference implementation focuses on the self-heating effect of a single-stage CMOS power amplifier. The complete approach is discussed in Wolf et al. (2010). The transistor-level model is depicted in Fig. 4 for a single device, as well as in Fig. 7 for a stacked-cascade single stage amplifier. Details on the transistor to transistor interaction due to thermal coupling is discussed in Schleyer et al. (2014). 


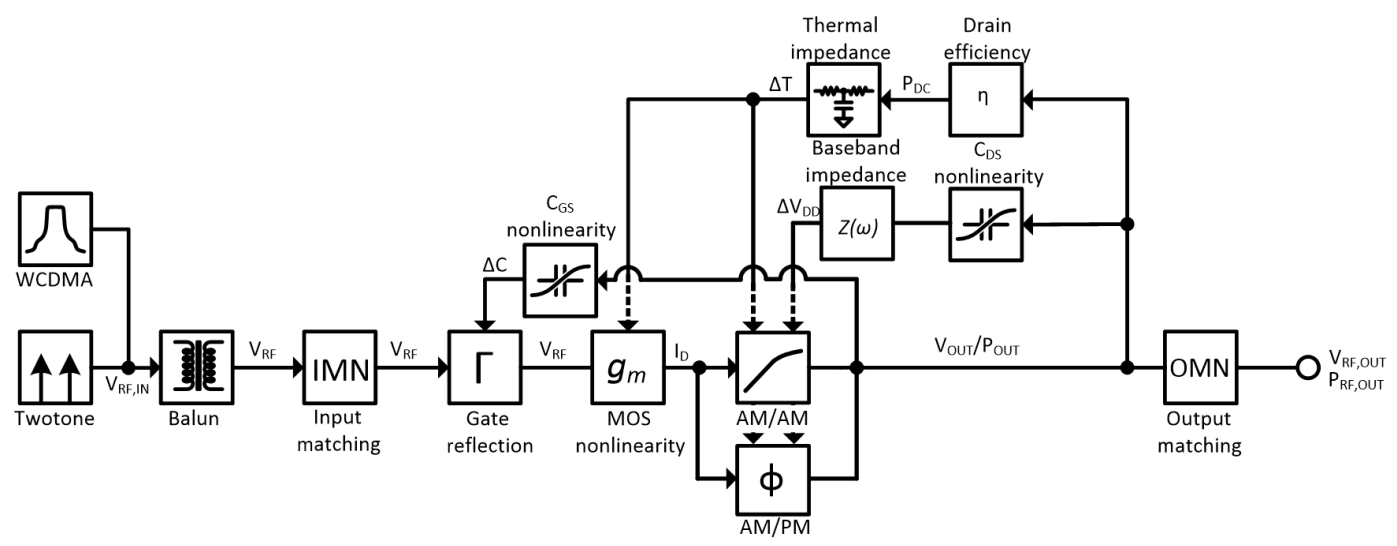

Figure 3. Detailed SystemC/AMS block level modelling for the parameter extraction.

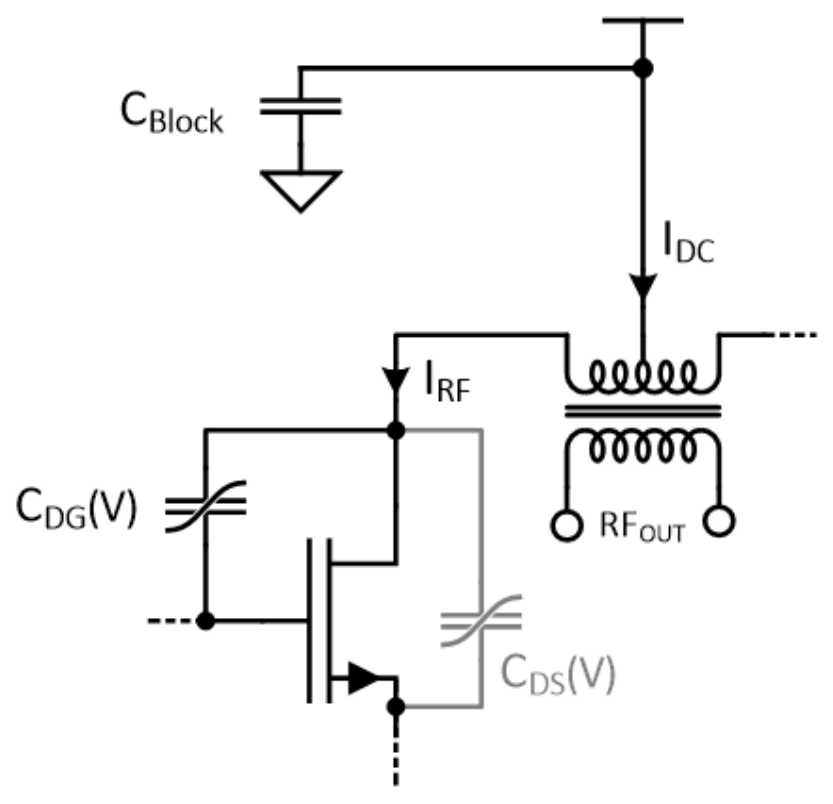

Figure 4. Band pass effect of the drain bias node.

\subsection{Root-cause analysis}

Using the proposed measurement concept the memory effect is scanned (measured and quantified) over envelop frequency (two tone spacing) and input amplitude variation. As compared to other measurement approaches which only focus on certain inter-modulation products (IMDs), the twotone method does not suffer from information loss. This is possible because the efficient modelling approach allows a full scan of a wide two-tone range, as well as for the full amplitude range in an affordable time and computation power. However, the measurement is significantly more effort. Therefore, an appropriate concept is introduced, to shorten the characterization procedure, as shown in Figs. 1 and 3. A detailed introduction into the automation and model

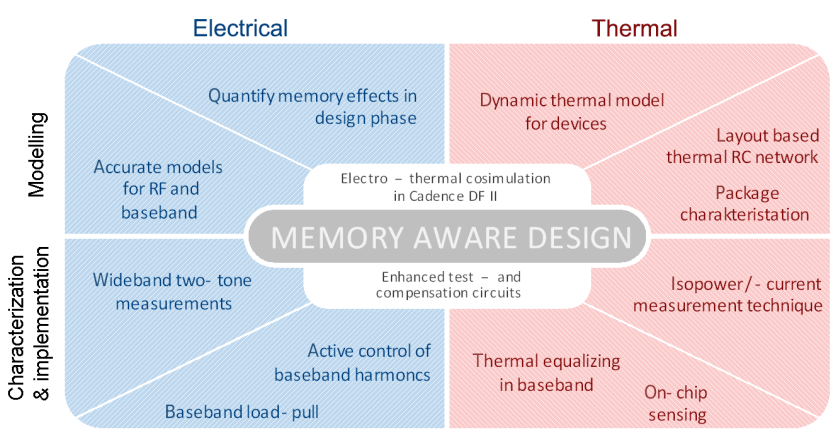

Figure 5. Memory-aware design concept.

verification is given in Schleyer et al. (2014). The algorithm processes the AM/AM and AM/PM time-domain trajectories, and applies calibration with chirp-signals (Wolf et al., 2009). The design framework is optimized for electrothermal co-design. By transferring the thermal behavior into an equivalent electrical model ( $\mathrm{RC}$ approach, thermal coevaluation), also electrical evaluation methods of circuit simulators are useable.

\subsection{Thermo-induced operating point effects}

Due to a nonlinear drain capacitance $C_{\mathrm{DS}}\left(V_{\mathrm{drain}}\right)$, the frequency response of the bias network causes a voltage drop at drain nodes (Fig. 4). This shifts and modulates the operation point of the transistor, which effects the gain. The effect is twofold - the gain variation results in a AM/AM hysteresis, and the varying $C_{\mathrm{DS}}\left(V_{\text {drain }}\right)$ causes a $\mathrm{AM} / \mathrm{PM}$ hysteresis. Hence, there is essentially an electrical bandpass effect, which also distorts the baseband impedance (Moreira et al., 2015). 


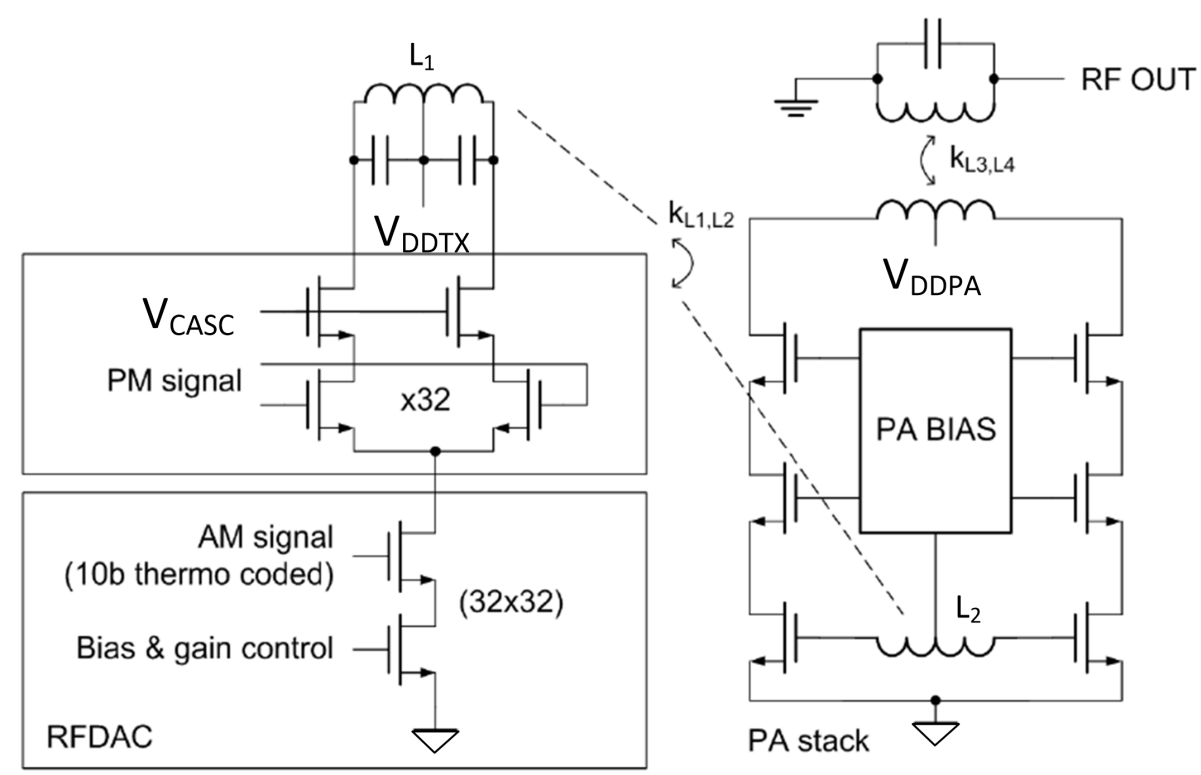

Figure 6. Connection of RFDAC and an integrated PA.

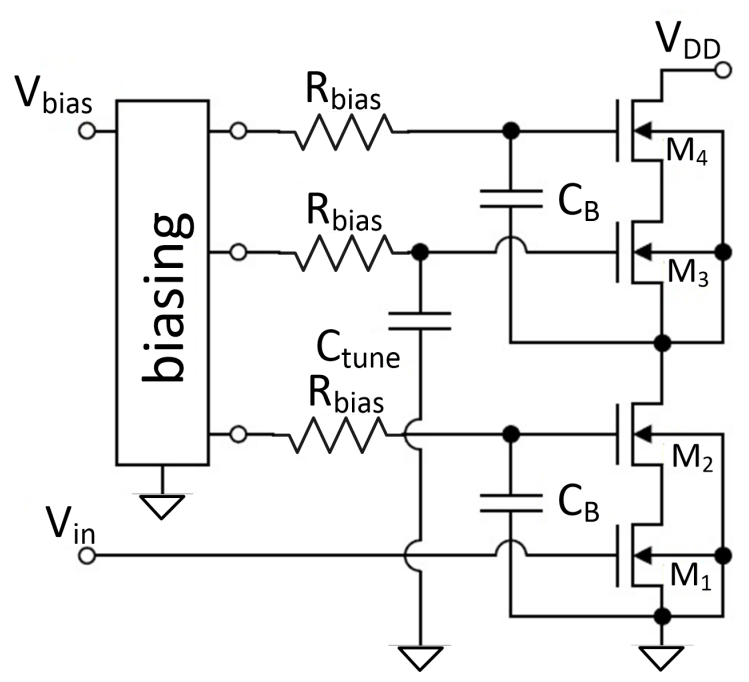

Figure 7. Stacked cascode circuit with dynamic biasing.

\subsection{Specification driven design methodology}

Figure 5 concludes the electro-thermal effects, and maps them to modelling as well as implementation concepts. A memory aware design certainly relies on the designer's experience, but coordinating the root-cause and design options in a structured and quantifiable manner inevitable for good performance results. As system specifications tend to higher carrier frequencies, as well as a high integration density, low $Q$-factors of passive components as well as the thermal interaction, e.g. in flip-chip packages, add design complexity and need to be optimized. In addition, appropriate biasing con- cepts, multi-stage feedback structures, and impedance transformation networks need to be optimized, as well as the bandwidth limitation to be understood.

Memory-aware specification allow more effective and reliable designs.

\section{Design example: dynamic biasing in CMOS RF power amplifiers}

Based on the root-cause analysis provided above, this section discusses two successful design approaches to compensate memory effects in power amplifiers. The discussion here focuses on the output stage of power amplifiers as they transform most power into heat.

\subsection{Connection of RFDAC and integrated PA}

In order to compensate for the thermally induced $C_{\mathrm{DS}}$ and bias point modulation the gate biasing needs to be adapted accordingly. A possible implementation is presented in Fig. 6 (Moreira et al., 2015). A RF digital-to-analog converter (RFDAC) is used to generate bias and gain control as well as an AM signal. In addition, a differential PM signal improves the dynamic bias tracking for reduced memory effect. Eventually the bias setting is RF-coupled to the power amplifier gate biasing circuit. The advantage of this approach is that the drain node of the PA transistors is at a constant level that implies zero drain impedance. Thus, the gain is only reduced by compression, but not by memory effects. Hence, also the baseband spectrum is improved.

As the circuit is implemented for mass production in $65 \mathrm{~nm}$ CMOS technology, a transistor stack is used to mit- 


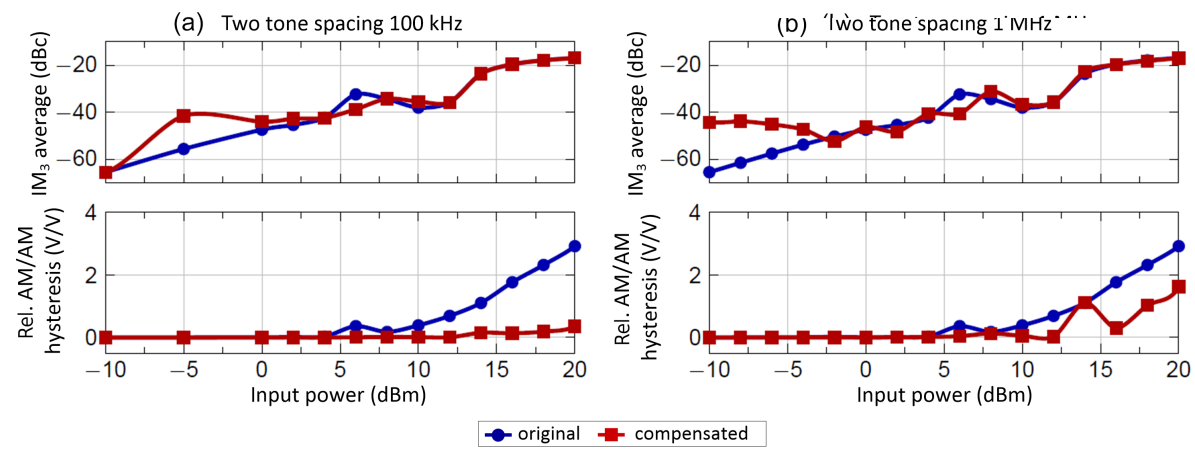

Figure 8. Improvement due to the compensation of memory effects. Flattening of the AM/AM line indicates the reduced dependency on the input signal amplitude (OIP1 equals $27 \mathrm{dBm}$, with a PAE of over $30 \%$ ).

igate device voltage stress. Due to this additional transistors, the design needs to be carefully optimized in order achieve a good trade-off between robustness to voltage stress and efficiency.

\subsection{Dynamic gate modulation (concept of VG2 modulation)}

Another design approach is to modulate the complete transistor well with a dynamic biasing, as illustrated in Fig. 7. A successful implementation of this approach is discussed in Leuschner et al. (2010) and Leuschner et al. (2011). The baseband impedance $Z_{\mathrm{BB}}$ is seen from the cascade load transistor into $V_{\mathrm{DD}}$ nodes. The drain impedance $Z_{M_{1}}$ is seen from $M_{1}$ into cascade. Thereby, the assumption is that the impedance $Z_{M_{1}}$ influences linearity and memory. Therefore, for large RF signals, the power amplifier goes into class-B operation, and the drain node of $M_{1}$ sees a varying impedance and thus the AM/AM and AM/PM behavior is altered. Hence, two direct implications are to avoid a phase shift between drain voltage and current, which can be achieved by a real-valued $Z_{\text {bias. }}$. Moreover, the termination of baseband (BB) components with a short circuit will reduce the $V_{\text {drain,BB }}$ component. The positive effect is a reduced PM hysteresis due to $C_{\mathrm{DS}}\left(V_{\mathrm{drain}, \mathrm{BB}}\right)$, and also a reduced $\mathrm{AM}$ hysteresis due to up-mixing of $V_{\text {drain,BB }}$.

The stacked cascode approach has different degrees of freedom. First, the bias network limited to one inductive element (transformer), second, the drain resistance seen from input transistor $M_{1}$ can be varied via the additional gate bias node. Third, overcompensation helps the correction of IM2 contribution. In addition, the overall IM3 can be real valued and hence symmetric.

\section{Performance results}

Applying the aforementioned design options from Sect. 3 together with the analysis methodology discussed in Sect. 2 allows already at design time the quantification of perfor- mance improvements. This is important for the system design as early insights are available regarding the need for or avoidance of additional digital linearization and pre-distortion measures.

In order to evaluate the performance improvement, a twotone excitation in the range of $100 \mathrm{kHz}$ and $1 \mathrm{MHz}$ is performed. The objective is the flattening of the AM/AM line as a result of the minimization of the dependency on input signal amplitude. As shown in Fig. 8, using dynamic bias point modulation and baseband injection can significantly reduce memory effects, which is visible by the reduced opening of graphs for compensated operation. Hence, using a standard peak-to-average-power-ratio (WCDMA), the improvement of AM/AM mismatch can be over $8 \%$ in average.

\section{Conclusions}

The root-cause analysis and design conclusions presented in this paper are shown to be relevant for future highperformance power amplifiers in radio front-ends. Analyzing and understanding the root-cause of the memory-effects and mapping them to design methodology leads to significant improvement, while additional complexity in the digital domain is avoided. Starting from an electro-thermal co-design approach with automated measurement setup, the electrical on operating point and parasitic device capacitances can be linked to the circuit behavior. The development of dynamic compensation methods revealed a stacked-cascode approach as well as a modulated-well technique as two potential candidates for the compensation of memory-effect by analog circuit design.

Data availability. Proprietary channel model from company was used and is not publicly available.

Competing interests. The authors declare that they have no conflict of interest. 
Acknowledgements. The authors gratefully acknowledge research funding provided by the DFG (German Research foundation), grant no. KL 918/8-1.

Edited by: J. Anders

Reviewed by: two anonymous referees

\section{References}

Bösch, W. and Gatti, G.: Measurement and simulation of memory effects in pre-distortion linearizers,IEEE Transactions on Microwave Theory and Techniques, 37.12, 1885-1890, https://doi.org/10.1109/22.44098, 1989.

Brunk, M. and Jüngel, A.: Self-heating in a coupled thermoelectric circuit-device model, J. Comput. Electron., 10, 163-178 https://doi.org/10.1007/s10825-010-0324-9, 2011.

Leuschner, S., Pinarello, S., Hodel, U., Mueller, J. E., and Klar, H.: A $31-\mathrm{dBm}$, high ruggedness power amplifier in $65-\mathrm{nm}$ standard CMOS with high-efficiency stacked-cascode stages, in: Radio Frequency Integrated Circuits Symposium (RFIC), IEEE, 395398, https://doi.org/10.1109/RFIC.2010.5477401, 2010.

Leuschner, S., Mueller, J.-E., and Klar, H.: A $1.8 \mathrm{GHz}$ wideband stacked-cascode CMOS power amplifier for WCDMA applications in $65 \mathrm{~nm}$ standard CMOS, in: Radio Frequency Integrated Circuits Symposium (RFIC), IEEE, 1-4, 2011.
Martens, J. and Noujeim, K.: IMD phase analysis at mm-wave frequencies, in: Microwave Symposium Digest (MTT), IEEE MTT-S International, https://doi.org/10.1109/MWSYM.2012.6259703, 2012.

Moreira, J., Kapfelsperger, B., and Pletzer, J.: A single-chip HSPA transceiver with fully integrated 3G CMOS power amplifiers, in: IEEE International Solid-State Circuits Conference - (ISSCC), Digest of Technical Papers, San Francisco, CA, 1-3, https://doi.org/10.1109/ISSCC.2015.7062976, 2015.

Schleyer, M., Leuschner, S., Baumgartner, P., Mueller, J.-E., and Klar, H.: An enhanced BSIM modeling framework for selfheating aware circuit design, Adv. Radio Sci., 12, 95-101, https://doi.org/10.5194/ars-12-95-2014, 2014.

Wolf, N., Mueller, J.-E., and Klar, H.: Identification of frequency dependent memory effects and the linearization of a CMOS PA for multiple standards, in: Radio and Wireless Symposium (RWS), IEEE, 598-601, https://doi.org/10.1109/RWS.2009.4957422, 2009.

Wolf, N., Müller, J.-E., and Klar, H.: Simple pre-distortion system for compensation of temperature dependent nonlinearity of power amplifiers, in: Radio and Wireless Symposium (RWS), IEEE, 152-155, https://doi.org/10.1109/RWS.2010.5434250, 2010.

Vuolevi, J., Rahkonen, T., and Manninen, J.: Measurement technique for characterizing memory effects in RF power amplifiers, in: Radio and Wireless Conference (RAWCON), IEEE, 195-198, https://doi.org/10.1109/RAWCON.2000.881888, 2000. 